\title{
Mechanical Power to Identify Human Performance for a Lower Limb Rehabilitation Robot
}

\author{
Trinnachoke Eiammanussakul ${ }^{\mathrm{a}}$ and Viboon Sangveraphunsiri ${ }^{\mathrm{b}, *}$ \\ Department of Mechanical Engineering, Faculty of Engineering, Chulalongkorn University, Bangkok \\ 10330, Thailand \\ E-mail: ${ }^{a}$ Trinnachoke.E@chula.ac.th, bViboon.S@chula.ac.th (Corresponding author)
}

\begin{abstract}
Rehabilitation robots usually provide trainings with a certain training modality and activity. The patient's performance measure such as mechanical power is also derived for those specific trainings. This article aims to demonstrate the implementation of our lower limb rehabilitation robot in sitting position for providing the training with games and to propose the derivation of the human mechanical power as a performance measure. The control algorithm for active exercise and the integration of the related software and hardware are also developed to offer proper environment for a game session. The derivation of the mechanical power of a human subject in the training is verified with active and passive cycling exercises. Three healthy subjects participate in the game sessions provided by the robot. It is found that the game sessions can provide the movement training with sufficient intensity. Moreover, the mechanical power obtained from the proposed method is able to identify the intensity of training tasks, human performance, and human attention in the training.
\end{abstract}

Keywords: Lower limb rehabilitation robot, stroke rehabilitation, mechanical power, human performance.

ENGINEERING JOURNAL Volume 23 Issue 4

Received 23 October 2018

Accepted 2 May 2019

Published 8 August 2019

Online at http://www.engj.org/

DOI:10.4186/ej.2019.23.4.91 


\section{Introduction}

In stroke rehabilitation, the training that facilitates effective recovery should consist of high intensity and repetition movements $[1,2]$. In the meantime, the patient should be motivated to actively engage in the training over a long period [3]. A conventional training that requires physiotherapists to manually assist the movement of a patient is expensive, labor-intensive, and low repetitive while the duration of the training session is short. In addition, intervention and evaluation technique are also different depending on theories and experience of physiotherapists $[1,4]$. Nowadays, robots have been developed for rehabilitation tasks extensively. The robots not only release physical burdens from physiotherapists so that intensity, repetition, and duration of the training could be increased, but also retrieve quantitative data during the therapy with equipped sensors [5] for objective evaluation of the patient's status [6]. Furthermore, various training modalities and activities can be selected to suit with the condition of the patient $[3,7]$. The robots can also be implemented with games and virtual reality systems to motivate the patient to actively participate in the training session [8-10]. Table 1 shows clinical results from trainings with games and virtual reality systems implemented by lower limb rehabilitation robots, namely, Rutgers Ankle [11], Anklebot [12], VRCTS [10]. Therefore, robots for stroke rehabilitation are promising tools to provide optimal recovery outcomes and enhance the productivity of physiotherapists [13].

To evaluate the ability of the patient, performance measures are obtained from multiple sensors equipped on the robots and the rehabilitation platforms. Mechanical power is one of performance measures widely used in research settings. It is usually derived specifically to a training activity. In cycling exercise, the mechanical power, which is used as one of the parameters to determine the cycling performance [14], is calculated from cadence and resistance torque applied to the motor of the ergometer. In sit-to-stand exercise, maximal power is determined from vertical ground reaction force with strain gauges installed under the rehabilitation platform during the rising phase. The sit-to-stand power is used to assess the muscle power of the patient [15]. During the exercise of the ankle joint with the Rutgers Ankle [16], data from force sensors mounted on the platform is used to indicate the patient's power. It is found that the improvement in patient's endurance corresponds to the power output of the affected ankle. In the trajectory tracking task with the MIT-MANUS [17], the power determined from interaction force in direction along and normal the target axis is obtained from torque sensors mounted on the motor shafts. It is used as a performance measure to quantify abilities to move and to aim the movement from the starting position towards the target.

This article presents the development of the controller for active exercise, and the integration of software and hardware for implementing game-based trainings with our lower limb rehabilitation robot in sitting position. The technique to evaluate the patient's performance is also devised so that the human mechanical power can be obtained regardless of training modality and activity.

This article is organized as follows. The configuration of the lower limb rehabilitation robot is described in Section 2. The control algorithm for active exercise is presented in Section 3. The implementation of games and the robot is shown in Section 4. In Section 5, the human mechanical power is derived and verified. Section 6 reports results from training sessions with games by a healthy subject. The conclusion of the article is in Section 7.

\section{Robot Description}

For a post-stroke patient that suffers from severe lower limb muscle weakness and cannot participate in a gait training session in a safe manner, the movement training in the sitting position is preferred. It is found that the outcomes of the training in sitting position can reduce motor impairments and translate to into improved walking ability $[10,11,18]$. The lower limb rehabilitation robot in our research is developed to provide physical therapy to the patient with this stage.

\subsection{Components of the Robot}

In Fig. 1, the robot consists of an exoskeleton, a counterbalance mechanism, and a control unit. The exoskeleton has 3 degrees of freedom at hip, knee, and ankle joints. The range of motion of the hip joint is $45^{\circ}$ flexion and $0^{\circ}$ extension. The movement of the knee joint is within $110^{\circ}$ flexion and $0^{\circ}$ extension. The ankle joint is able to move in the range of $20^{\circ}$ dorsiflexion and $45^{\circ}$ plantar flexion. There are mechanical stoppers at the ends of the joint movement range to ensure that the robot joints always stay within human's 
biological ranges of motion. Each joint of the exoskeleton is actuated by a brushless servo motor through a cable transmission mechanism. 400W motors are chosen as actuators for hip and knee joints while a 200W motor powers the ankle joint. The transmission ratios of the cable transmission mechanism are 57.8:1, 15:1, and 15:1 for hip, knee, and ankle joint respectively. The exoskeleton joint is designed so that it can provide enough assistive torque to a patient and still be backdrivable. The thigh, shank, and foot segments of the exoskeleton are telescopic so that the length can be adjusted to fit with a wearer's leg. Velcro straps are used to fasten a human leg to the exoskeleton at each segment. The exoskeleton (excluding the hip joint transmission mechanism that is mounted on the control unit) weighs 4.96 kilograms.

Table 1. Clinical results of lower limb robotic rehabilitation with games and virtual reality systems.

\begin{tabular}{lccccc}
\hline Robot & $\begin{array}{c}\text { Training } \\
\text { Modality }\end{array}$ & Training Activity & $\begin{array}{c}\text { Movement } \\
\text { Frequency } \\
\text { (rpm) }\end{array}$ & $\begin{array}{c}\text { Training } \\
\text { Period }\end{array}$ & Clinical Outcome \\
\hline $\begin{array}{l}\text { Rutgers } \\
\text { Ankle }\end{array}$ & $\begin{array}{c}\text { Active } \\
\text { resistive }\end{array}$ & $\begin{array}{c}\text { Ankle dorsiflexion/ } \\
\text { plantar flexion, } \\
\text { Ankle } \\
\text { inversion/eversion }\end{array}$ & 6.53 & $\begin{array}{c}4 \text { weeks } \\
(12 \text { sessions })\end{array}$ & $\begin{array}{r}\text { Improvements in } \\
\text { muscle strength } \\
\text { and walking speed }\end{array}$ \\
\hline Anklebot & $\begin{array}{c}\text { Active } \\
\text { assistive }\end{array}$ & $\begin{array}{c}\text { Ankle dorsiflexion/ } \\
\text { plantar flexion, } \\
\text { Ankle }\end{array}$ & 16 & $\begin{array}{c}6 \text { weeks } \\
\text { inversion/eversion }\end{array}$ & $\begin{array}{c}\text { Improvements in gait } \\
\text { speed, endurance, } \\
\text { and standing balance }\end{array}$ \\
\hline VRCTS & Active & Cycling & 59.83 & $\begin{array}{c}4 \text { weeks } \\
(10 \text { sessions })\end{array}$ & $\begin{array}{c}\text { Improvements in } \\
\text { pedal force symmetry } \\
\text { and standing balance }\end{array}$ \\
\hline
\end{tabular}

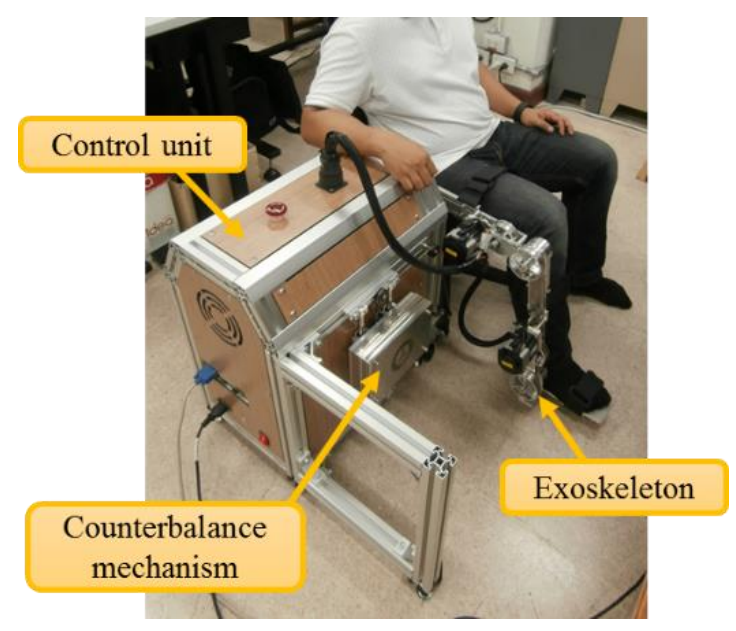

Fig. 1. Lower limb rehabilitation robot for the training in sitting position.

The counterbalance mechanism is designed to decrease the torque requirement of the actuator of the exoskeleton's hip joint. The mechanism has a $12-\mathrm{kg}$ counterweight with linear bearings that allows vertical movement along guide rods mounted on the control unit. The counterweight is linked to the thigh segment of the exoskeleton with a cable. The counterbalance moment about the hip joint, which is generated by the counterweight, changes according to the angular position of the thigh segment of the exoskeleton. With this design, the counterbalance torque can be generated up to $20.7 \mathrm{~N}$.m. The reduction of torque requirement implies that the robot joint can be designed with a smaller motor and lower transmission ratio which leads to lower reflected inertia. Therefore, the hip joint of the exoskeleton has better backdrivability and safety in human-robot interaction [19]. However, there is no counterbalance mechanism to reduce the torque requirement of the actuators of knee and ankle joints for simplicity of the robot design. 
The control unit is used as a platform for mounting the exoskeleton and the counterbalance mechanism. It also contain a DC power supply, a computer unit, a data acquisition card, motor drivers, an emergency stop button, and other electronic components. More details of the robot development are referred to our previous publication [20].

\subsection{Dynamic Model of the Exoskeleton}

In Fig. 2(a), hip, knee, and ankle joints are linked by the exoskeleton segments. The structure can be simplified as a three-revolute-joint serial robot moving in the sagittal plane as shown in the schematics in Fig. 2(b). Point $\mathrm{O}$ represents the hip joint which is fixed on a stationary base (a control unit). Point $\mathrm{A}$ and $\mathrm{B}$ are the knee joint and the ankle joint whereas point $\mathrm{C}$ is the end of the foot segment. The coordinate frame has the origin at point $\mathrm{O}$ and the direction of frame axes are selected as shown in the figure. $L_{0}, L_{1}$, and $L_{2}$ are the overall length of thigh, shank, and foot segments of the exoskeleton while $l_{0}, l_{1}$, and $l_{2}$ are the distance between the center of gravity of each link and the proximal joint. $\theta_{0}, \theta_{1}$, and $\theta_{2}$ are angles of hip, knee and ankle joint measured with respect to the neutral sitting position (illustrated by dashed lines). Masses of the exoskeleton segments are abbreviated as $m_{0}, m_{1}$, and $m_{2}$. The gravitational acceleration $(\mathrm{g})$ has the same direction as the positive y-axis. According to [21], the dynamic model of the exoskeleton can be written as:

$$
\mathbf{B}_{\mathrm{e}}(\theta) \ddot{\theta}+\mathbf{C}_{\mathrm{e}}(\theta, \dot{\theta}) \dot{\theta}+\mathrm{G}_{\mathrm{e}}(\theta)=\tau_{\mathrm{e}}-\tau_{\mathrm{int}}
$$

where frictions are neglected. $\mathbf{B}_{\mathrm{e}}$ is the inertia matrix of the exoskeleton. $\mathbf{C}_{\mathrm{e}}(\boldsymbol{\theta}, \dot{\boldsymbol{\theta}}) \dot{\boldsymbol{\theta}}$ represents the matrix of torque due to Coriolis and centripetal effects while the matrix $\boldsymbol{\theta}=\left[\begin{array}{lll}\theta_{0} & \theta_{1} & \theta_{2}\end{array}\right]^{\mathrm{T}}$ and $\dot{\boldsymbol{\theta}}=\left[\begin{array}{lll}\dot{\theta}_{0} & \dot{\theta}_{1} & \dot{\theta}_{2}\end{array}\right]^{\mathrm{T}}$. $\mathbf{G}_{\mathbf{e}}$ is the matrix of the gravitational load due to the exoskeleton weight. With known mass, length, and location of center of gravity of each segment, the matrices $\mathbf{B}_{\mathrm{e}}, \mathbf{C}_{\mathrm{e}}$, and $\mathbf{G}_{\mathrm{e}}$ can be derived mathematically. $\tau_{\mathrm{e}}$ is the actuator torque at the exoskeleton joints while $\tau_{\text {int }}$ is the torque generated from the interaction between the exoskeleton and the environment (which is a human subject in this case).

\section{Control Algorithm for Active Exercises}

This lower limb rehabilitation robot is able to perform various therapeutic exercises. Control algorithms for passive, active assistive, and active resistive exercises are referred to our previous publication [22]. In this article, the control algorithm of the active exercise is proposed to be implemented by the lower limb rehabilitation robot in game-based trainings. Active exercise is suitable for a patient with some muscle strength to move the paretic leg against gravity by himself [3]. Therefore, the control algorithm for active exercise aims to allow voluntary movement by a patient without any assistance or resistance by the robot.

The control algorithm for active exercise as shown in Fig. 3 consists of 2 cascaded loops. In the outer control loop, the reference torque $\left(\tau_{\mathrm{r}}\right)$ is the summation of the desired torque $\left(\tau_{\mathrm{d}}\right)$ and gravity compensation torque $\left(\tau_{\mathrm{g}}\right)$ as follows:

$$
\tau_{\mathrm{r}}=\tau_{\mathrm{d}}+\tau_{\mathrm{g}} .
$$

This implies that the actuator will only exert torque to compensate for gravitational load due to the exoskeleton weight. With the counterbalance mechanism, the gravity compensation torque of the hip joint is reduced by the amount of the counterbalance moment generated by the mechanism. In the torque control loop, a PI controller is selected to minimize torque error. The output of this PI controller is the desired joint velocity $\left(\dot{\theta}_{\mathrm{i}, \mathrm{d}}\right)$ which is the reference signal for the inner control loop where the other PI controller is implemented in order to generate control signal to a motor driver. Motor position $\left(\theta_{\mathrm{m}}\right)$ is measured by an encoder mounted on the motor shaft. The joint position $\left(\theta_{\mathrm{j}}\right)$ is then estimated by dividing motor position by transmission ratio $(\mathrm{N})$. Joint velocity $\left(\dot{\theta}_{\mathrm{j}}\right)$ is the time derivative of the joint position. Joint torque of the 
exoskeleton $\left(\tau_{\mathrm{c}}\right)$ is obtained from the motor torque, which is measured by a motor driver, multiplied by the transmission ratio. The proof of stability and passivity of the controller can be found in our publication [20].

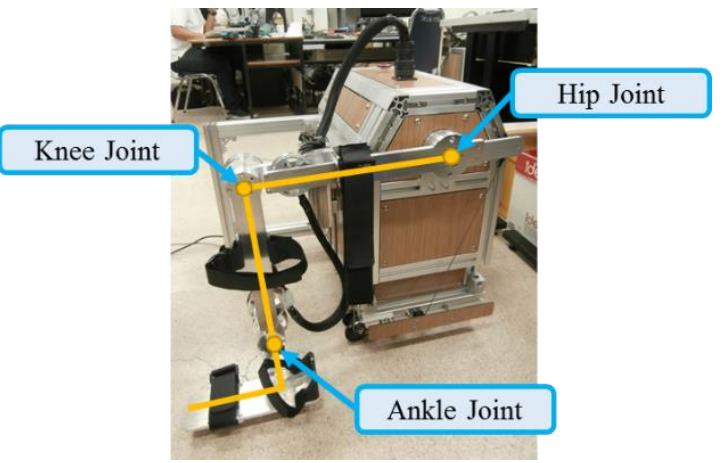

(a)

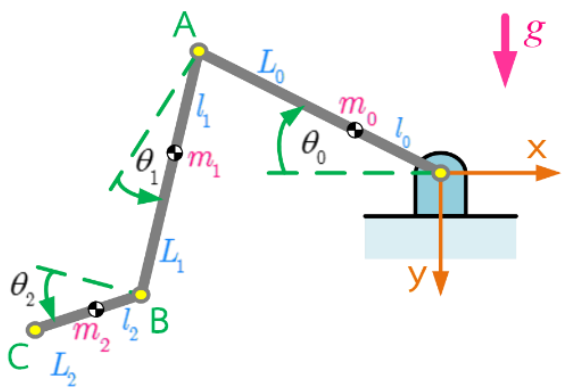

(b)

Fig. 2. The exoskeleton structure: (a) Joint location; (b) Simplified schematics.

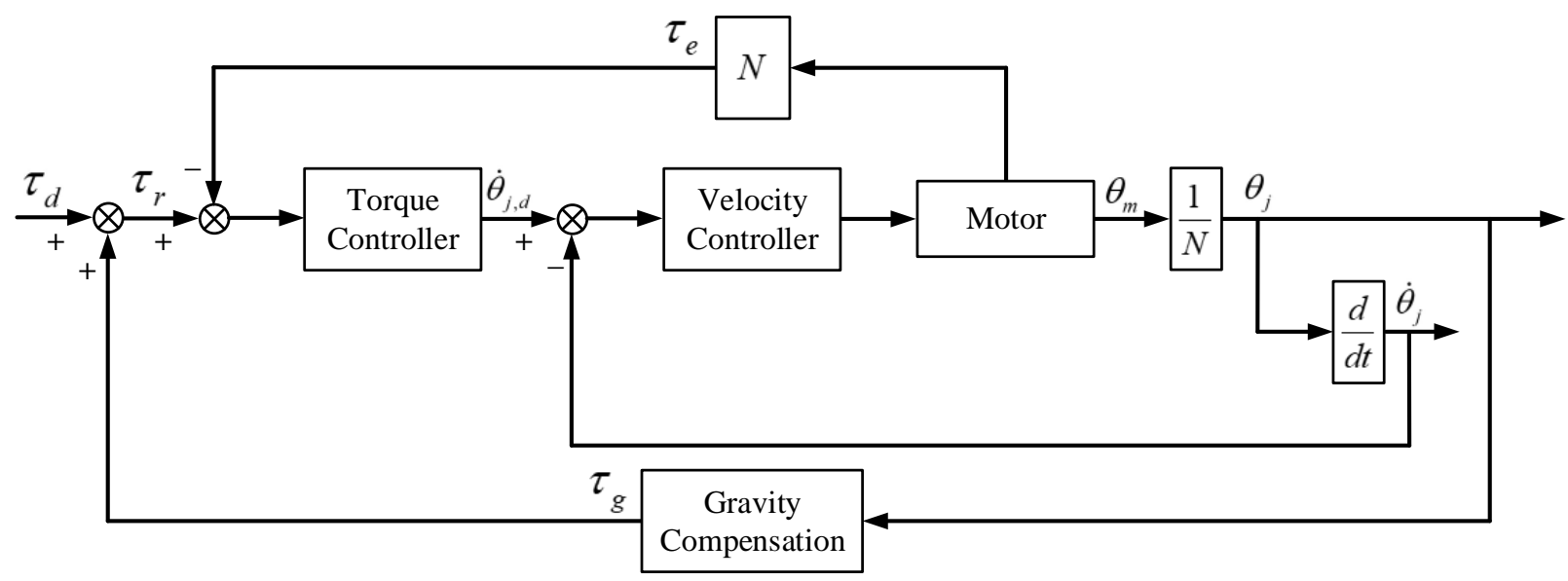

Fig. 3. Block diagram of control algorithm for active exercise.

Unlike other training modalities, active exercise does not assist or resist the movement of a patient. The desired torque is set to zero:

$$
\tau_{\mathrm{r}}=\tau_{\mathrm{g}}
$$

\section{System Implementation}

\subsection{Software and Hardware Communication}

The system is operated on a computer running Microsoft Windows. There are two applications for implementing a robot to play a game. Firstly, the robot control application is an executable application based on the Microsoft Foundation Class (MFC) Library. This application includes a user interface to set up operation mode, starts/stops the robot operation, display robot operating status, and implement the robot controller. The second application is for playing games. In this research, BlueStacks App Player is chosen. This free-downloadable software enables Android applications (including games) to run on a Windowsoperated computer. The applications run on BlueStacks can be controlled with a mouse click, a keyboard stroke, and an external touch pad input. Android applications can be downloaded and installed in the computer. Afterwards, they can be played on Bluestacks either online or offline.

According to Fig. 4, the robot control application receives data from a data acquisition card installed in a computer. If the program detects the movement of the exoskeleton, it will synthesize a virtual keystroke to 
BlueStacks (which runs as the foreground application). By setting a desired keystroke for controlling the game in the keyboard control user interface of BlueStacks as shown in Fig. 5, the user can move the exoskeleton to trigger an action in the game.

\subsection{Exoskeleton Movement for Triggering an Action in the Bluestacks}

Before the start of a game, the user must select the desired joint to send a virtual keystroke and trigger an action in the robot control application. Fig. 6 shows the joint movements that can be selected for sending a single command to a game: hip flexion, knee extension, or ankle dorsiflexion. During the game session, if the desired joint moves in the desired direction with velocity more than the desired threshold, the robot control application will determine that the movement occurs intentionally. On the contrary, the movement that occurs opposite to the preset direction or has the magnitude of velocity below the threshold is considered as an unintended action or noise that might originated from the differentiation of angular position with respect to time. The value of the threshold is obtained empirically to ensure that the noise is neglected and only intentional movements are detected.

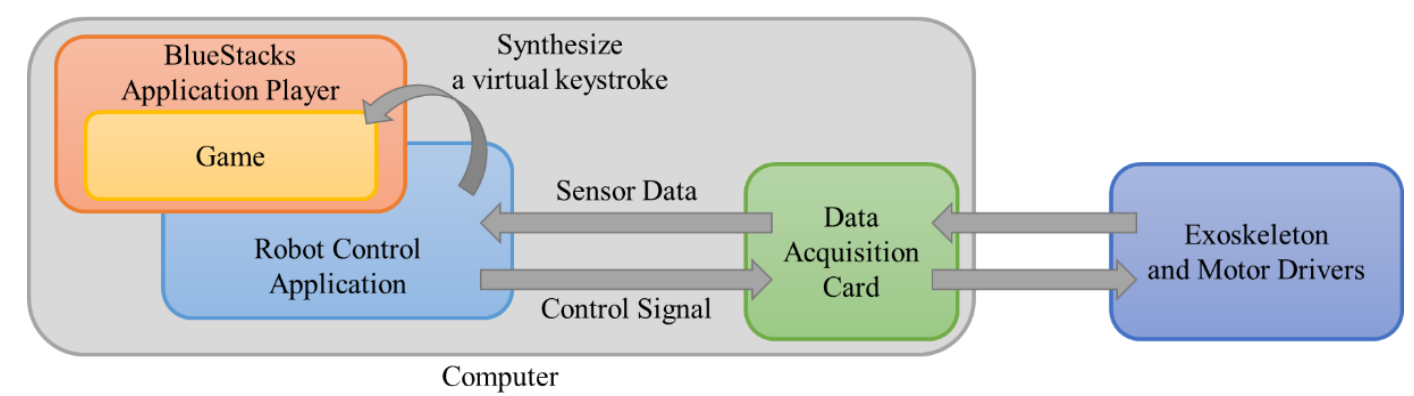

Fig. 4. Software and hardware communication.

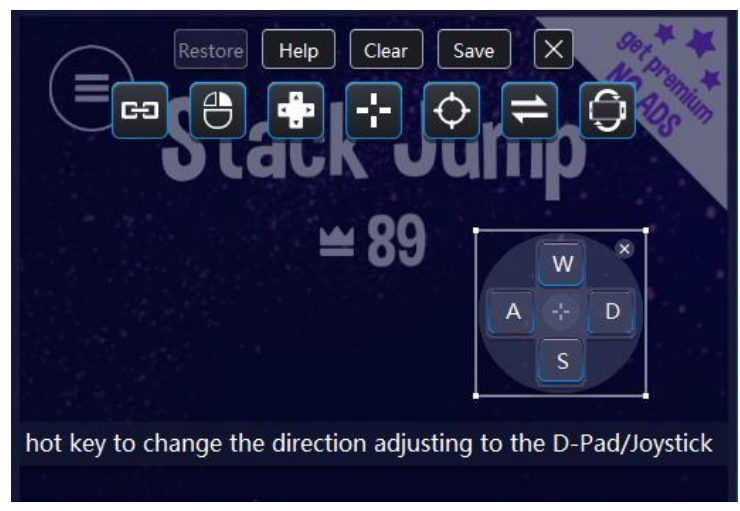

Fig. 5. Keyboard control user interface in BlueStacks.

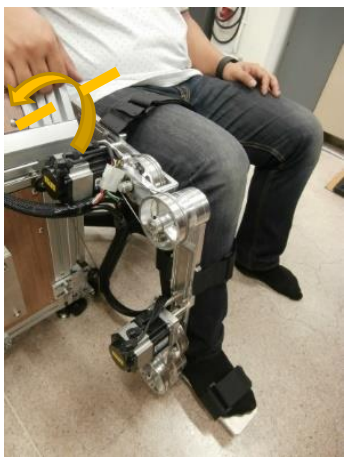

(a)

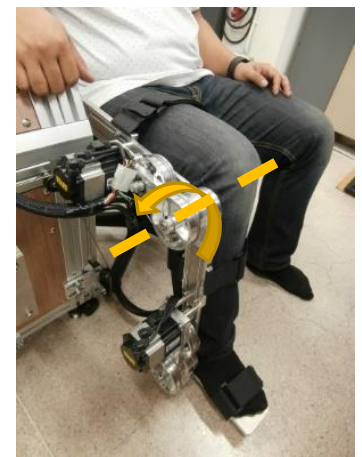

(b)

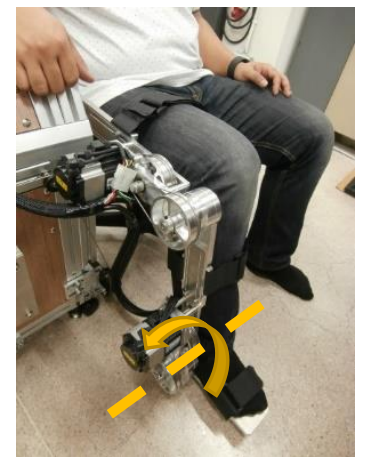

(c)

Fig. 6. Movement of the exoskeleton for sending a virtual keystroke and triggering an action in a game in direction of (a) hip flexion; (b) knee extension; (c) ankle dorsiflexion. 


\section{Human Mechanical Power}

\subsection{Human Mechanical Power Derivation}

To obtain simple mathematical model of a human leg, several assumptions are made. First of all, a human leg has three rigid segments (thigh, shank, and foot) and three simple hinge joints (hip, knee, and ankle joints). In addition, each segment of the exoskeleton is fastened to a human's leg, so the movement of the human leg is restricted only on the sagittal plane and kinematic data (position, velocity, and acceleration) of a human leg and the exoskeleton is the same. Moreover, torque generated by elastic and damping properties of hip, knee, and ankle joints are neglected since the elastic torque becomes significant only near the end of the range of motion [23-24] while low damping coefficients [24] and joint velocities produce small amount of resistive torque. Taking these assumptions into consideration, the model of a human leg is similar to (1) but with the opposite direction of $\tau_{\text {int }}$ according to the Newton's third law:

$$
\mathbf{B}_{\mathrm{h}}(\boldsymbol{\theta}) \ddot{\boldsymbol{\theta}}+\mathbf{C}_{\mathrm{h}}(\boldsymbol{\theta}, \dot{\theta}) \dot{\boldsymbol{\theta}}+\mathbf{G}_{\mathrm{h}}(\boldsymbol{\theta})=\tau_{\mathrm{h}}+\tau_{\text {int }}
$$

where $\mathbf{B}_{\mathrm{h}}$ is the inertia matrix of the human leg, $\mathbf{C}_{\mathrm{h}}(\boldsymbol{\theta}, \dot{\boldsymbol{\theta}}) \dot{\boldsymbol{\theta}}$ is the matrix of torque due to Coriolis and centripetal effects, $\mathbf{G}_{\mathrm{h}}$ is the matrix of the gravitational load due to weight of the human leg, $\tau_{\mathrm{h}}$ is the vector of joint torque generated by human, and $\tau_{\text {int }}$ is the vector of interaction torque between the exoskeleton and the human whose magnitude is the same as that in (1). With known height $(\mathrm{H})$ and weight $(\mathrm{W})$ of a human subject, parameters of each segment can be estimated from the anthropomorphic data [25] as shown in Table 2. These data are used to derive $\mathbf{B}_{\mathrm{h}}, \mathbf{C}_{\mathrm{h}}$, and $\mathbf{G}_{\mathrm{h}}$ in the same manner as those of the exoskeleton.

The dynamic model of the whole system (the exoskeleton and a human leg) is derived from the summation of (1) and (2). The interaction torque is viewed as the internal torque which is not presented in the model of the overall system:

$$
B_{e}(\theta) \ddot{\theta}+C_{e}(\theta, \dot{\theta}) \dot{\theta}+G_{c}(\theta)+B_{h}(\theta) \ddot{\theta}+C_{h}(\theta, \dot{\theta}) \dot{\theta}+G_{h}(\theta)=\tau_{e}+\tau_{h}
$$

Therefore, the human torque can be calculated from the following equation:

$$
\tau_{\mathrm{h}}=\mathbf{B}_{\mathrm{e}}(\theta) \ddot{\theta}+\mathrm{C}_{\mathrm{e}}(\theta, \dot{\theta}) \dot{\theta}+\mathrm{G}_{\mathrm{e}}(\theta)+\mathrm{B}_{\mathrm{h}}(\theta) \ddot{\theta}+\mathrm{C}_{\mathrm{h}}(\theta, \dot{\theta}) \dot{\theta}+\mathrm{G}_{\mathrm{h}}(\theta)-\tau_{\mathrm{e}}
$$

The mechanical power of the human subject at the $\mathrm{i}^{\text {th }}$ joint is the product between the human torque and the joint velocity:

$$
\mathrm{P}_{\mathrm{h}}^{\mathrm{i}}=\tau_{\mathrm{h}}^{\mathrm{i}} \dot{\theta}^{\mathrm{i}}
$$

For the mechanical power of the exoskeleton at the $i^{\text {th }}$ joint, it is calculated with measured joint torque and the joint velocity:

$$
\mathrm{P}_{\mathrm{e}}^{\mathrm{i}}=\tau_{\mathrm{e}}^{\mathrm{i}} \dot{\theta}^{\mathrm{i}}
$$

Table 2. Anthropomorphic data of a human leg.

\begin{tabular}{lcccr}
\hline Segment & $\begin{array}{c}\text { Segment } \\
\text { Length (L) }\end{array}$ & $\begin{array}{c}\text { Segment } \\
\text { Weight (W) }\end{array}$ & $\begin{array}{c}\text { Location of } \\
\text { Center of } \\
\text { Mass }\end{array}$ & $\begin{array}{r}\text { Radius of Gyration } \\
\text { about the Center of } \\
\text { Mass of the } \\
\text { Segment }\end{array}$ \\
\hline Thigh & $0.53 \mathrm{H}$ & $0.1 \mathrm{~W}$ & $0.433 \mathrm{~L}$ & $0.475 \mathrm{~L}$ \\
\hline Shank & $0.246 \mathrm{H}$ & $0.0465 \mathrm{~W}$ & $0.433 \mathrm{~L}$ & $0.302 \mathrm{~L}$ \\
\hline Foot & $0.039 \mathrm{H}$ & $0.0145 \mathrm{~W}$ & $0.5 \mathrm{~L}$ & $0.323 \mathrm{~L}$ \\
\hline
\end{tabular}




\subsection{Validation of the Derived Mechanical Power with Active Assistive Training Modality}

The experiment is conducted in order to verify that the mechanical powers of human and the exoskeleton derived in the previous section are able to identify the amount of effort of each power source contributing to the movement. The cycling exercise by the robot is selected to train a subject with active assistive modality. With this training modality, the subject is instructed to move his lower limbs to track a predefined trajectory in sagittal plane. The active assistive control algorithm, proposed in our previous publication [20], allows the subject to dominate the movement if the position deviation is low while the exoskeleton provides assistance to the patient as much as needed to complete the movement. Ideally, if the patient is strong enough such that he can track the reference trajectory by himself, the exoskeleton will only follow the human movement without any intervention. Conversely, if the patient is too weak and cannot move on his own, the exoskeleton will provide assistive torque to move the subject's leg along the reference trajectory.

Three volunteered healthy subjects (without history of neurological disorder) participates in the experiment. Their demographic data are provided in Table 3. The subjects participated in two sessions of cycling exercise using active assistive training modality. Each session consists of ten movement repetitions. In the first training session, the subjects track the cycling movement actively so that the assistance provided by the exoskeleton is minimized. In another training session, the subjects act passively by relaxing their lower limbs so that the movement is achieved solely by the exoskeleton assistance. The mechanical powers of the subjects and the exoskeleton at each joint are calculated from (3) and (4).

Computed mechanical powers of subject 2 and the exoskeleton are illustrated in Fig. 7. It can be seen that their mechanical powers are not constant in one movement cycle and different when compared to those in other cycles. Mechanical power can be positive, negative, or zero. According to (3) and (4), components of mechanical power are joint torque and joint velocity. If joint torque is applied in the same direction as the joint velocity, mechanical power becomes positive, and vice versa. Therefore, the subject and the exoskeleton always apply effort to affect the movement regardless of the sign of mechanical power.

According to Fig. 7 (a), during active cycling, the mechanical powers of the subject are high whereas the exoskeleton exerts small amount of mechanical power. It can be implied that the subject put effort into tracking the reference trajectory while the exoskeleton only compensates for its dynamics and gravitational load. On the contrary, the mechanical powers of the subject in Fig. 7 (b) are lower than those of the exoskeleton in passive cycling and the subject in active cycling. This indicates that the subject has less effort in passive cycling while the exoskeleton generates more mechanical power in order to support the subject's leg and track the reference trajectory. Therefore, the mechanical powers deriving from section 5.1 can reflect how much effort each power source (the subject and the exoskeleton) applies to influence the resultant movement. For further analysis, the root-mean-square (RMS) value is used to represent the mechanical power of the entire the training.

Fig. 8 shows the RMS mechanical powers of the three subjects and the exoskeleton at each joint in active and passive cycling. In Fig. 8 (a), it can be seen that each subject exerts different amount of mechanical power during active and passive cycling. This implies that they track the reference trajectory by focusing on different joint, i.e., subject 1 has the RMS mechanical powers at hip and knee joints more than the exoskeleton while subject 2 exerts higher mechanical power compared with the exoskeleton only at the hip joint unlike subject 3 that has the RMS mechanical power at the knee joint higher than that of the exoskeleton. However, according to the RMS mechanical power in passive cycling as shown in Fig. 8 (b), all subjects exert less effort than the exoskeleton at every joint. It can also be noticed that the RMS mechanical powers of the subjects in active cycling are higher than those in passive cycling. Therefore, the RMS mechanical power is able to quantify the performance of the subject

Table 3. Demographic data of participants.

\begin{tabular}{lccr}
\hline Subject & $\mathbf{1}$ & $\mathbf{2}$ & $\mathbf{3}$ \\
\hline Gender & Male & Female & Male \\
\hline Age (year) & 30 & 45 & 25 \\
\hline Height (cm) & 168 & 165 & 171 \\
\hline Weight (kg) & 65 & 53 & 61 \\
\hline
\end{tabular}



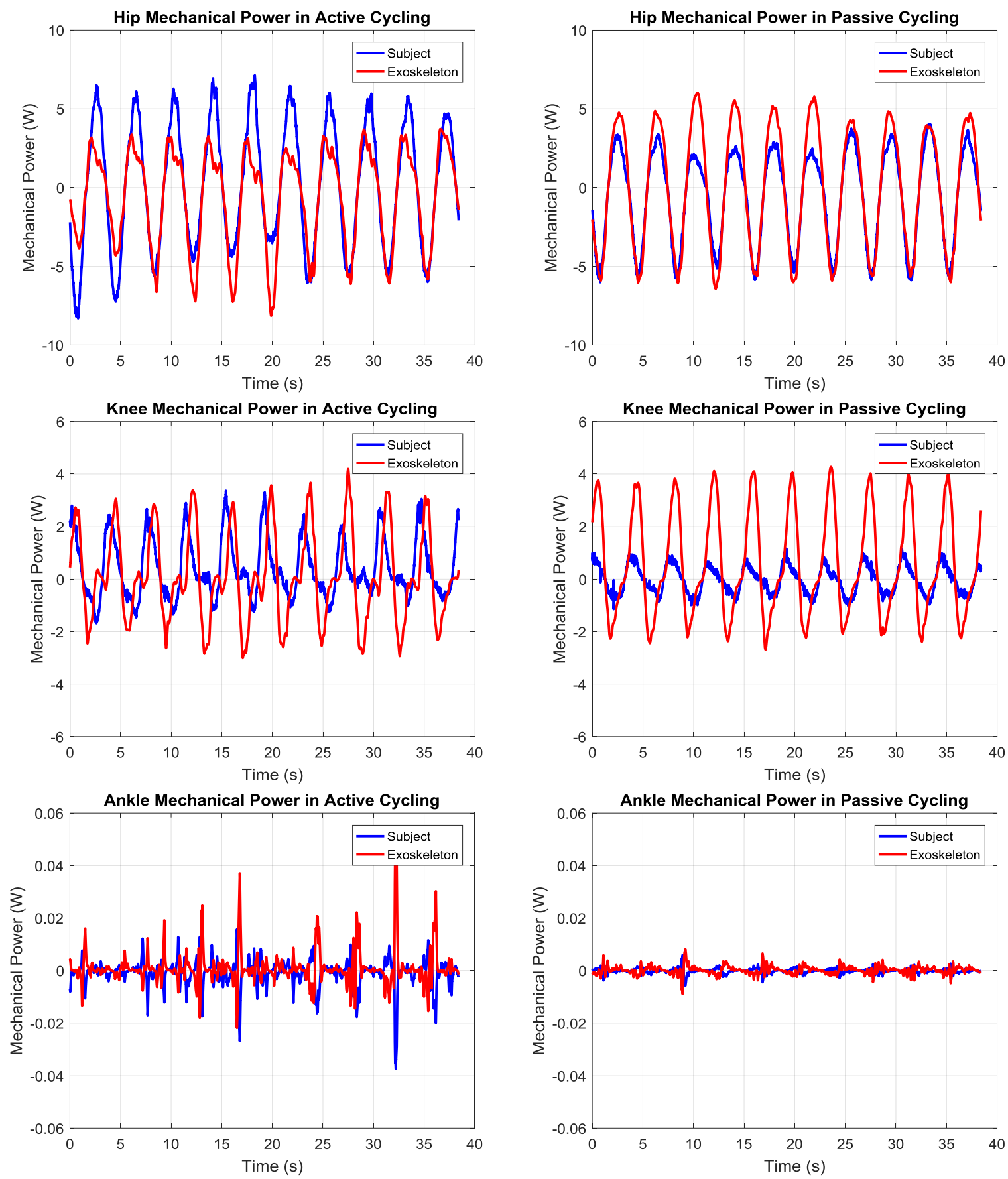

(a)

(b)

Fig. 7. Mechanical power of subject 2 during (a) active cycling; (b) passive cycling.

\section{Results from Game Sessions}

To demonstrate the operation of the system and human performance while playing games, two Android games are chosen to be played by healthy subjects in Table 3 with the exoskeleton as shown in Fig. 9. The first game as shown in Fig. 10 (a) is "Stack Jump" which is an arcade game developed by VOODOO. The goal of the game is to build a stack tower high enough to complete a level or to make a high score. The player must trigger an input to the game so that the cartoon character in the game could jump on a stack block that moves from left or right side towards the center of the screen with different speed. Next stack block move at higher height so that the character could jump on them and achieve a taller stack tower. The game ends if the character fails to jump and collides with the moving stack. The game is scored by the number of successful 
jumps (the height of the stack tower). The second game is "Armor" which is an arcade game developed by Pixels On Fire Games. The goal of the game is to hit the target circle where multiple layers of armors orbit around it. A player must trigger an input to the game in order to shoot a ball from the bottom of the screen towards the target circle. Some armors are able to be smashed by the shooting balls while the others must be avoided otherwise the game ends. The player may need to shoot several balls to get rid of some blocking armors and then hit the target circle in order to proceed to the next level.
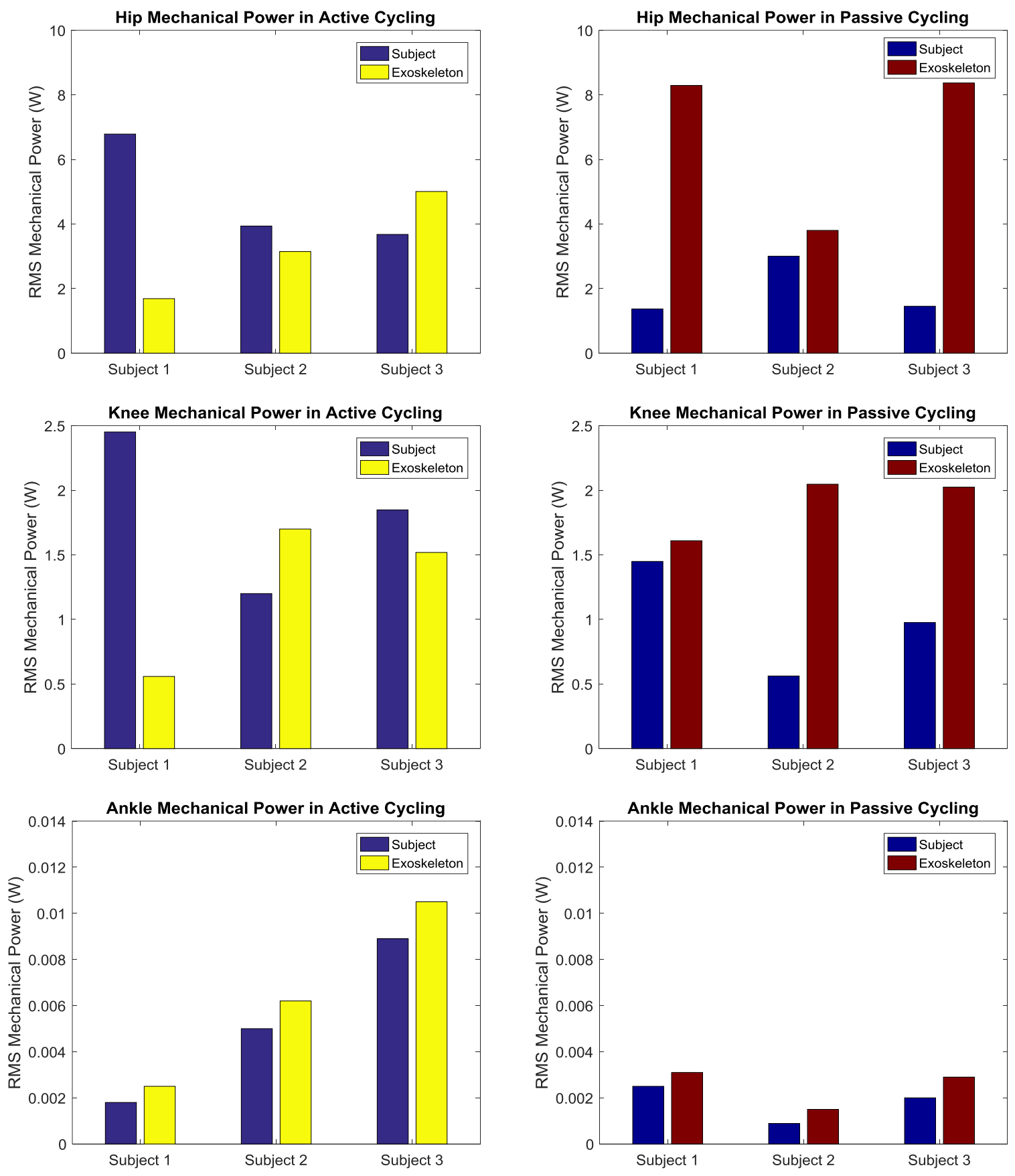

(a)

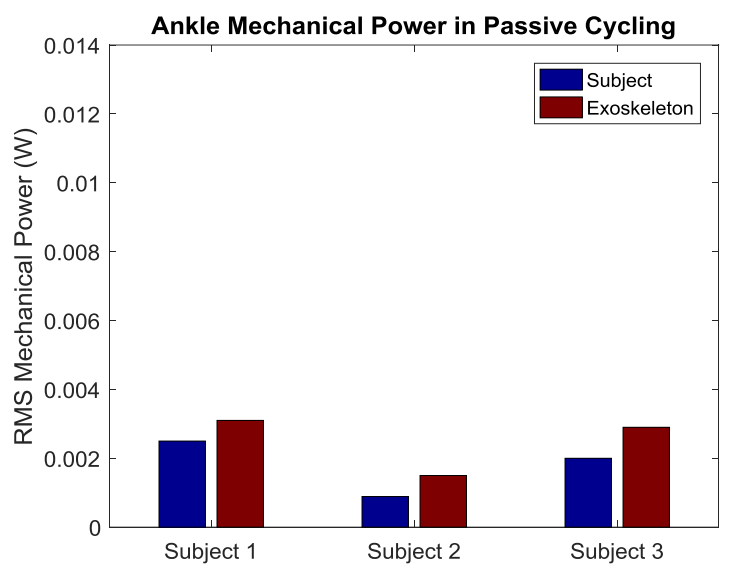

(b)

Fig. 8. RMS mechanical power of subjects and the exoskeleton during (a) active cycling; (b) passive cycling. 


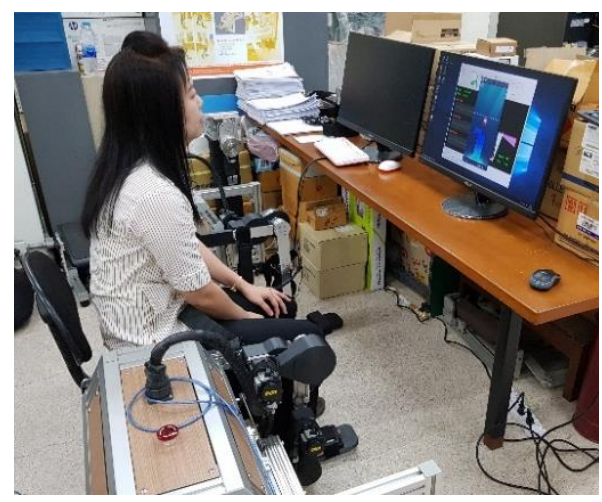

Fig. 9. Game session by a healthy subject with the robot.

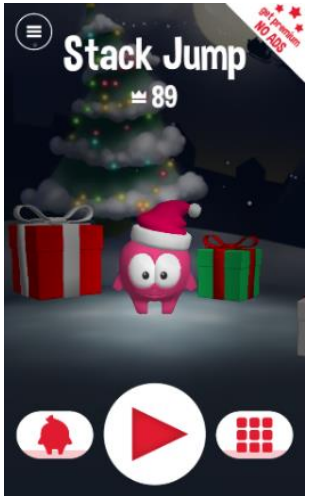

(a)

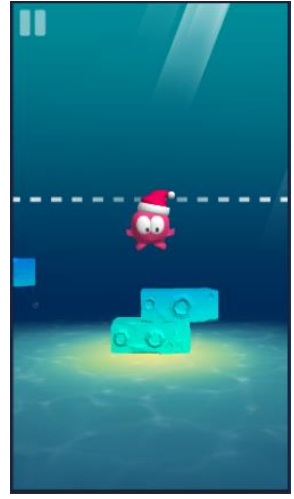

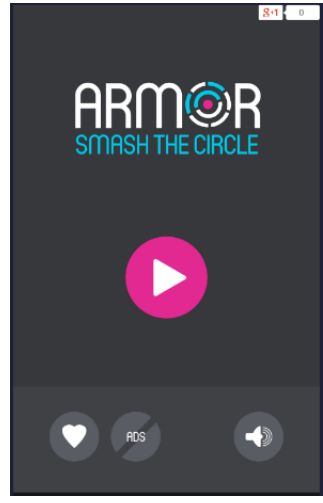

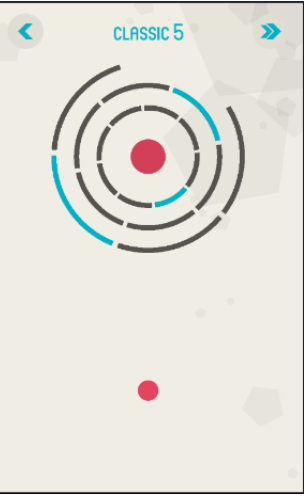

(b)

Fig. 10. Android games (a) Stack Jump (b) Armor.

\subsection{Training with Different Games by the Same Joint}

In this experiment, the subjects play each game for 10 minutes. The ankle dorsiflexion movement and the velocity threshold of $0.3 \mathrm{rad} / \mathrm{s}$ are selected to produce a triggering signal to control the games. The detection of the desired ankle movement triggers the jumping of the character in "Stack Jump" and the shooting of a ball in "Armor". If the game is over, the subjects continue the game until the game session ends.

As noticed from Fig. 11, the amount of movement of every subject increases uniformly in "Stack Jump" as opposed to that in "Armor" which sometimes increases with lower or higher rate. There are several factors that might affect the frequency of the movement. Firstly, different nature of these games might force or prevent the subjects to move the ankle joint constantly. In "Stack Jumps", the player is urged to move so that the character could jump on the incoming stack and avoid collision. However, in "Armor", the player has to wait for a chance to shoot a ball into the target circle or smash breakable armors while avoiding shooting on forbidden armors. Therefore, the timing condition of these games influences the amount of movement in the game sessions. Another factor that might relate to the amount of movement of the subjects is their attention during the game sessions. The subject that feels excited while playing games tends to move frequently whereas the subject that has low interest in playing the games is prone to move slowly. Thus, when using the robot in clinical settings, the amount of movement could be used as an index to determine the attention of the subject during a training session. It also suggests whether a patient needs more motivation or the difficulty level of the game has to be adjusted to suit with the ability of the patient.

According to Table 4, the total amount of movement is independent of games and subjects. Subject 1 moves while playing "Armor" more than in "Stack Jump" whereas subject 2 plays "Stack Jump" with higher repetition than that in "Armor" and subject 3 has a similar amount of movement in both games. 


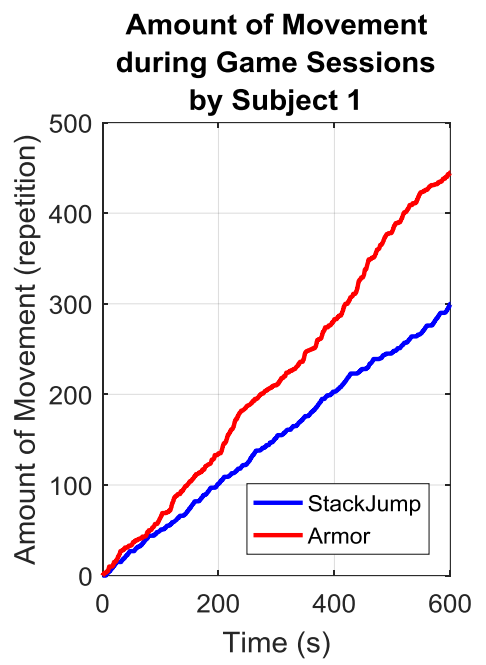

(a)

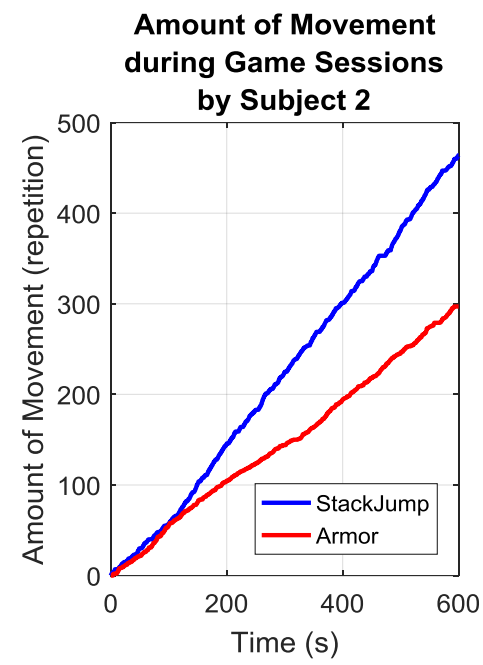

(b)

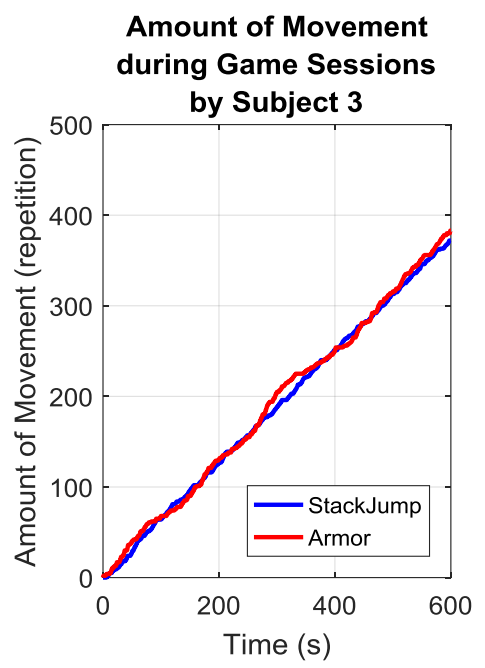

(c)

Fig. 11. Amount of movement of the ankle joint during "Stack Jump" and "Armor" sessions of (a) subject 1; (b) subject 2; (c) subject 3

Table 4. Results from playing "Stack Jump" and "Armor" with the ankle joint of the subjects.

\begin{tabular}{lccccr}
\hline & $\begin{array}{c}\text { Total Amount } \\
\text { of Movement } \\
\text { (repetition) }\end{array}$ & $\begin{array}{c}\text { Movement } \\
\text { Frequency } \\
\text { (rpm) }\end{array}$ & $\begin{array}{c}\text { RMS } \\
\text { Velocity } \\
(\mathbf{m} / \mathbf{s})\end{array}$ & $\begin{array}{c}\text { RMS } \\
\text { Torque } \\
\text { (N.m) }\end{array}$ & $\begin{array}{r}\text { RMS } \\
\text { Mechanical } \\
\text { Power (W) }\end{array}$ \\
\hline Subject1 & & & & & \\
- Stack Jump & 300 & 30.0 & 0.200 & 0.170 & 0.043 \\
- Armor & 445 & 44.5 & 0.140 & 0.168 & 0.029 \\
\hline $\begin{array}{l}\text { Subject 2 } \\
\text { - Stack Jump }\end{array}$ & 464 & 46.4 & 0.389 & & \\
- Armor & 297 & 29.7 & 0.120 & 0.152 & 0.127 \\
\hline $\begin{array}{l}\text { Subject 3 } \\
\text { - Stack Jump }\end{array}$ & 372 & 37.2 & 0.278 & 0.168 & 0.018 \\
- Armor & 383 & 38.3 & 0.137 & 0.167 & 0.061 \\
\hline
\end{tabular}

It also found that no subject moves less than another. For example, subject 1 has the lowest amount of movement in "Stack Jump" session among the subjects but subject 1 moves with the highest repetition in "Armor" session. Moreover, movement frequencies, which are the averaged amount of movement in one minute, of every subject in all sessions are higher than those in the training with the Rutgers Ankle and the anklebot as shown in Table 1. In other words, playing games with the robot can help subjects to move their limbs with high repetitions. Therefore, the training with games by the robot might be applied to stroke patients to improve their clinical outcomes. Furthermore, it can be noticed from Table 4 that all subjects exert higher mechanical power in "Stack Jump" more than in "Armor". The reason is that the subjects move the ankle joint each time in "Stack Jump" with higher velocity and torque than in "Armor". Therefore, the nature of games not only affects the amount of movement and attention of the players but also the intensity of the movement.

\subsection{Training with the Same Game by Different Joints}

The subjects also plays "Armor" with the hip joint and the knee joint for 10 minutes in each session (with the velocity threshold of $0.3 \mathrm{rad} / \mathrm{s}$ ). The result from the game session controlled with the ankle joint is obtained from the experiment in section 6.1 in order to compare how the controlling joint affects the training.

As seen from Fig. 12, slopes of the amount of movement in each training session are not constant which corresponds to the findings from the section 6.1. This is due to the nature of the game that requires proper timing to trigger actions. 
According to Table 5, even though each subject plays the game with different amount of movements. It can be noticed that each subject plays the game with hip, knee, or ankle joints with similar amount of movement in the training sessions. Therefore, the joint selected to trigger the signal to the game can be chosen arbitrarily since is does not influence the amount of movement in the training session. Moreover, it is found that the RMS mechanical power of each subject while playing the game with the hip joints is the highest followed by the knee joints and the ankle joints. By inspecting the components of the mechanical power, although the RMS velocities of the knee joint sessions are the highest, the RMS torque of the hip joints of the subjects are extremely high compared to those of the knee joints. As a result, their products (mechanical powers) have the highest values in the training sessions played with the hip joints. In the training sessions with the ankle joints, small magnitude of velocity and torque produce low mechanical power. This shows that for the movement trainings by using different joints, the RMS mechanical power is able to quantify the intensity of the tasks because it considers both velocity and torque.

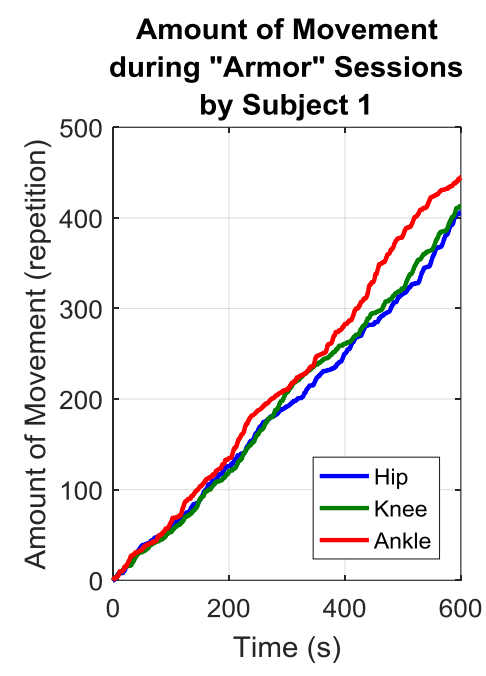

(a)

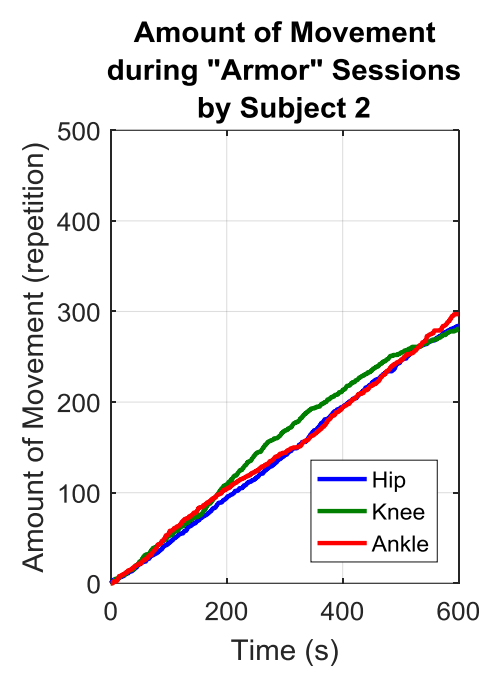

(b)

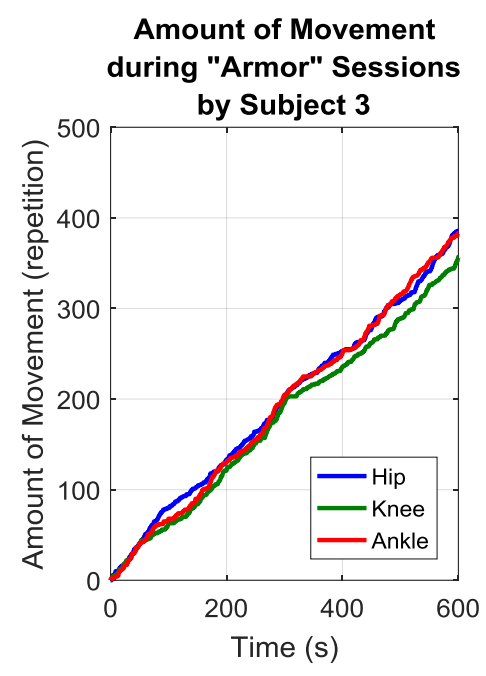

(c)

Fig. 12. Amount of movement of hip, knee, and ankle joints during "Armor" sessions of (a) subject 1; (b) subject 2; (c) subject 3 .

Table 5. Result from playing "Armor" with hip, knee, and ankle joints of the subjects.

\begin{tabular}{cccccc}
\hline & $\begin{array}{c}\text { Total Amount } \\
\text { of Movement } \\
\text { (repetition) }\end{array}$ & $\begin{array}{c}\text { Movement } \\
\text { Frequency } \\
\text { (rpm) }\end{array}$ & $\begin{array}{c}\text { RMS } \\
\text { Velocity } \\
\text { (m/s) }\end{array}$ & $\begin{array}{c}\text { RMS } \\
\text { Torque } \\
\text { (N.m) }\end{array}$ & $\begin{array}{c}\text { RMS } \\
\text { Mechanical } \\
\text { Power (W) }\end{array}$ \\
\hline Subject1 & & & & & \\
- Hip & 406 & 40.6 & 0.159 & 31.276 & 5.001 \\
- Knee & 413 & 41.3 & 0.355 & 4.781 & 2.041 \\
- Ankle & 445 & 44.5 & 0.140 & 0.168 & 0.029 \\
\hline Subject 2 & & & & & \\
- Hip & 284 & 28.4 & 0.136 & 23.764 & 3.247 \\
- Knee & 281 & 28.1 & 0.334 & 2.197 & 1.025 \\
- Ankle & 297 & 29.7 & 0.120 & 0.127 & 0.018 \\
\hline Subject 3 & & & & & \\
- Hip & 386 & 38.6 & 0.161 & 28.246 & 4.604 \\
- Knee & 357 & 35.7 & 0.289 & 2.847 & 1.152 \\
- Ankle & 383 & 38.3 & 0.137 & 0.167 & 0.028 \\
\hline
\end{tabular}




\section{Conclusion}

Robotic trainings with games have shown the potential to recover stroke patients in clinical trials. In this article, the control algorithm for active exercise and the implementation of related software and hardware are developed so that the lower limb rehabilitation robot in sitting position could provide game training sessions. Moreover, the characteristics of the exoskeleton that moves together with the human's leg is applied in order to obtain the human mechanical power at each joint for any training activity and modality. It is shown that the derived mechanical power is able to identify the performance of the human subject in active and passive cycling exercises. In the training sessions with games ("Stack Jump" and "Armor") controlled by a single joint (hip, knee, or ankle joint), the movement frequencies are higher than those from some clinical tests reported in previous research. Therefore, the game-based training with the lower limb rehabilitation robot is promising to provide exercise with sufficient intensity for a patient to gain positive clinical outcomes. Moreover, it is found that the human mechanical power in these game sessions is able to identify the intensity of the tasks, the human performance at each joint, as well as, the attention of the subject during the training session. Therefore, the robot providing game sessions would help monitoring the progress of patient recovery and the evaluation of the performance of a patient.

In the next stage of the research, other kinds of movement to trigger a signal to the game should be included in order to target on other aspects of the training, for example, triggering a signal when a human subject reaches the joint limit which is similar to active range of motion exercise. Other types of action in a game should also be investigated in order to diversify a variety of games and motivate a patient to participate in the training.

\section{Acknowledgement}

This research work is a part of the project supported by Ratchadapisek Somphot Fund for Postdoctoral Fellowship, Chulalongkorn University, the Research Pyramid and Chula Research Scholar project, the Second Century Research Policy of Chulalongkorn University, 2016-2018, and the National Research University Project, Office of Higher Education Commission, Project no. WCU-013-HR-57 and CU-59-005-IC.

\section{References}

[1] M. Babaiasl, S. H. Mahdioun, P. Jaryani, and M. Yazdani, "A review of technological and clinical aspects of robot-aided rehabilitation of upper-extremity after stroke," Disabil Rehabil Assist Technol, vol. 11, no. 4, pp. 263-280, Jan. 2016.

[2] H. Vallery, J. Veneman, E. van Asseldonk, R. Ekkelenkamp, M. Buss, and H. van Der Kooij, "Compliant actuation of rehabilitation robots," IEEE Robot Autom Mag, vol. 15, no. 3, pp. 60-69, Oct. 2008.

[3] E. Akdogan and M. A. Adli, "The design and control of a therapeutic exercise robot for lower limb rehabilitation: Physiotherabot," Mechatronics, vol. 21, no. 3, pp. 509-522, Apr. 2011.

[4] W. Meng, Q. Liu, Z. Zhou, Q. Ai, B. Sheng, and S. Xie, "Recent development of mechanisms and control strategies for robot-assisted lower limb rehabilitation," Mechatronics, vol. 31, pp. 132-145, Oct. 2015.

[5] I. Díaz, J. J. Gil, and E. Sánchez, "Lower-limb robotic rehabilitation: literature review and challenges," Journal of Robotics, vol. 2011, Sep. 2011.

[6] X. Zhang, Z. Yue, and J. Wang, "Robotics in Lower-Limb Rehabilitation after Stroke," Behav Neurol, vol. 2017, Jun. 2017.

[7] A. Basteris, S. M. Nijenhuis, A. H. A. Stienen, J. H. Buurke, G. B. Prange, and F. Amirabdollahian, "Training modalities in robot-mediated upper limb rehabilitation in stroke: A framework for classification based on a systematic review," J Neuroeng Rehabil, vol. 11, no. 111, Jul. 2014.

[8] J. C. Perry, J. Audureu, F. I. Cavallaro, J. Veneman, S. Carmien, and T. Keller, "Effective game use in neurorehabilitation: user-centered perspectives," in Handbook of Research on Improving Learning and Motivation through Educational Games: IGI Global, 2010, ch. 32, pp. 683-725.

[9] K. Laver, S. George, S. Thomas, J. E. Deutsch, and M. Crotty, "Virtual reality for stroke rehabilitation," Stroke, vol. 43, no. 2, pp. e20-e21, Nov. 2012. 
[10] C. Yin, Y. H. Hsueg, C. Y. Yeh, H. C. Lo, and Y. T. Lan, "A virtual reality-cycling training system for lower limb balance improvement," BioMed Res Int, vol. 2016, Jan. 2016.

[11] J. E. Deutsch, A. S. Merians, S. Adamovich, H. Poizner, and G. C. Burdea, "Development and application of virtual reality technology to improve hand use and gait of individuals post-stroke," Restor Neurol Neurosci, vol. 22, no. 3-5, pp. 371-386, Feb. 2004.

[12] J. L. Chang, R. Y. Lin, M. Saul, P. J. Koch, H. I. Krebs, B. T. Volpe, "Intensive seated robotic training of the ankle in patients with chronic stroke differentially improves gait," NeuroRehabilitation, vol. 41, no. 1, pp. 61-68, Jul. 2017.

[13] A. Jayaraman, S. Burt, and W. Z. Rymer, "Use of lower-limb robotics to enhance practice and participation in individuals with neurological conditions," Pediatr Phys Ther, vol. 29, pp. S48-S56, Jul. 2017.

[14] S. I. Lin, C. C. Lo, P. Y. Lin, and J. J. J. Chen, "Biomechanical assessments of the effect of visual feedback on cycling for patients with stroke," J Electromyogr Kinesiol, vol. 22, no. 4, pp. 582-588, Aug. 2012.

[15] P. Y. Chen, S. H. Wei, W. L. Hsieh, J. R. Cheen, L. K. Chen, and C. L. Kao, "Lower limb power rehabilitation (LLPR) using interactive video game for improvement of balance function in older people," Arch Gerontol Geriatr, vol. 55, no. 3, pp. 677-682, Jul. 2012.

[16] J. E. Deutsch, J. Latonio, G. C. Burdea, and R. Boian, "Post-stroke rehabilitation with the Rutgers Ankle system: a case study," Presence, vol. 10, no. 4, pp. 416-430, Aug. 2001.

[17] H. I. Krebs, N. Hogan, M. L. Aisen, and B. T. Volpe, "Robot-aided neurorehabilitation," IEEE Trans Rebabil Eng, vol. 6, no. 1, pp. 75-87, Mar. 1998.

[18] L.W. Forrester, A. Roy, A. Krywonis, G. Kehs, H. I. Krebs, and R. F. Macko, "Modular ankle robotics training in early sub-acute stroke: a randomized controlled pilot study," Neurorehabil Neural Repair, vol. 28, no. 7, pp. 678-687, Sep. 2014.

[19] B. Rooks, "The harmonious robot," Ind robot, vol. 33, no. 2, pp. 125-130, Mar. 2006.

[20] T. Eiammanussakul and V. Sangveraphunsiri, "A lower limb rehabilitation robot in sitting position with a review of training activities," J Healthc Eng, vol. 2018, Apr. 2018.

[21] B. Siciliano, L. Sciavicco, L. Villano, and G. Oriolo, "Dynamics," in Robotics: Modelling, Planning and Control: Springer, 2009, ch. 7, pp. 247-302.

[22] T. Eiammanussakul and V. Sangveraphunsiri,"Lower limb rehabilitation robot in sitting position for various therapeutic exercises," in Proceedings of the 9th International Conference on Bioinformatics and Biomedical Technology, 2017, pp. 112-116.

[23] B. Hwang and D. Jeon, "A method to accurately estimate the muscular torques of human wearing exoskeletons by torque sensors," Sensors, vol. 15, no. 4, pp. 8337-8357, Apr. 2015.

[24] A. Silder, B. Whittington, B. Heiderscheit, and D. G. Thelen, "Identification of passive elastic joint moment-angle relationships in the lower extremity," J Biomech, vol. 40, no. 12, pp. 2628-2635, Mar. 2007.

[25] D. A. Winter, "Anthropometry" in Biomechanics and Motor Control of Human Movement, $4^{\text {th }}$ ed. New Jersey: John Wiley \& Sons, 2009, ch. 4, pp. 82-106. 